\title{
Sarecycline Demonstrated Reduced Activity Against Representative Bacterial and Fungal Microflora Commonly Present in the Human Gastrointestinal Tract
}

Mahmoud A. Ghannoum 1,2 Janet Herrada1, Ahmed Gamal1,2, Lisa Long ${ }^{1,2}$, Thomas S. McCormick2, and Ayman Grada ${ }^{3}$

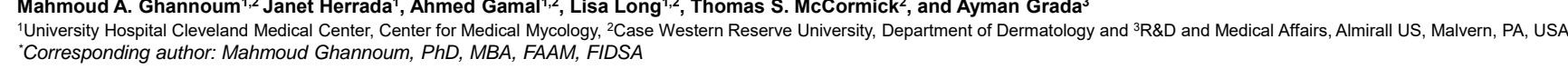

\section{Objective}

Evaluate the effect of sarecycline, a narrow spectrum antibiotic, compared to minocycline, a broad-spectrum antibiotic, against a panel of microorganisms that reflect the diversity of the gut microbiome using in vitro minimum inhibitory concentration (MIC) testing and timekill kinetic assays.

\section{Methods}

1. Chose representative bacterial and fungal strains found in the Healthy Gut

\section{Perform Antimicrobial Susceptibility Testing} Minimum inhibitory concentration (MIC) testing was performed using modified Clinical Laboratory Standards Institute methodology

\section{Establish Growth Curves}

E. coli and Candida tropicalis were selected as representative of aerobic bacteria and yeast, respectively. While Lactobacillus paracasei and Bifidobacterium adolescentis were selected as representative of anaerobic bacteria that colonize the gut.
Table 1. Susceptibility Testing Results for sarecycline and minocycline Against Healthy Gut Microbes $(\mu \mathrm{g} / \mathrm{mL}, \mathrm{n}=28$ )

\begin{tabular}{|c|c|c|c|c|c|}
\hline Phylum & Genus & Species & Sarecycline & Minocycline & $\begin{array}{l}\text { Fold Difference in } \\
\text { Mic }\end{array}$ \\
\hline Actinobacteria & Bifidobacterium & Bifidobacterium adolescentis & 1 & 1 & 1 \\
\hline Actinobacteria & collinsella & Collinsella aerofaciens & 1 & 0.5 & 2 \\
\hline Actinobacteria & Eggerthella & Eggerthella lenta & 1 & 0.5 & 2 \\
\hline Actinobacteria & Actinomycetales & Propionibacterium freudenrefichii & 8 & 1 & 8 \\
\hline Bacteroridetes & Bacteroides & Bacteroides caccae & 8 & 0.25 & 32 \\
\hline Bacteroidetes & Bacteroides & $\mid \begin{array}{l}\text { Bacterorides fragailis } \\
\text { enterotoxigenic (ET) }\end{array}$ & 2 & 4 & 0.5 \\
\hline Bacteroidetes & Bacteroides & Bacteroides fragilis nontoxigenic & 1 & 0.25 & 4 \\
\hline Bacteroidetes & Bacteroides & Bacteroides ovatus & 0.5 & 0.5 & 1 \\
\hline Bacteroidetes & Bacteroides & Bacteroides thetaiotaomicron & 0.25 & 0.125 & 2 \\
\hline Bacteroidetes & Bacteroides & Bacteroides uniformis & 2 & 0.5 & 4 \\
\hline Bacteroidetes & Bacteroides & Bacteroides vulgatus & 0.125 & 0.016 & 7.8 \\
\hline Bacteroidetes & Bacteroides & Bacteroides xylanisolvens & 1 & 0.25 & 4 \\
\hline Bacteroidetes & Bacteroides & Bifidobacterium subtile Biavati & $>8$ & 8 & Not Determined \\
\hline Bacteroidetes & Odoribacter & odoribacter splanchnicus & 8 & 4 & 2 \\
\hline Bacteroidetes & Parabacteroides & Parabacteroides distasonis & 8 & 2 & 4 \\
\hline Bacteroidetes & Parabacteroides & Parabacteroides merdae & 0.06 & 0.016 & 3.8 \\
\hline Firmicutes & Blautia & Blautia obeum & 1 & 0.5 & 2 \\
\hline Firmicutes & Clostridium & Clostridium bolteae & 4 & 0.5 & 8 \\
\hline Firmicutes & clostridium & Clostridium ramosum & 2 & 0.06 & 33.3 \\
\hline Firmicutes & clostridium & Clostridium saccharolyticum & 2 & 2 & 1 \\
\hline Firmicutes & Dorea & Dorea formicigenerans & 0.25 & 0.06 & 4.2 \\
\hline Firmicutes & Eubacterium & Eubacterium eligens & $>8$ & 4 & Not Determined \\
\hline Firmicutes & Lactobacillus & Lactobacillus paracasei & 1 & 0.25 & 4 \\
\hline Proteobacteria & Escherichia & Escherichia coli ial1 & 16 & 8 & 2 \\
\hline Sac fungi & Candida & Candida albicans & 32 & 16 & 2 \\
\hline Sac fungi & candida & Candida glabrata & 32 & 32 & 1 \\
\hline Sac fungi & candida & Candida parapsilosis & 32 & 16 & 2 \\
\hline Sac fungi & Candida & Candida tropicalis & 16 & 16 & 1 \\
\hline
\end{tabular}

$\square$ Higher fold difference indicates lower sarecycline activity

Compared to minocycline, sarecycline showed significantly less antimicrobial activity against: - 10 of 12 isolates from the Bacteroidetes phylum

- 3 out of 4 isolates from Actinobacteria phylum

- 5 of 7 isolates from the Firmicutes phylum, E. coll

- Propionibacterium freudenreichii ( $\geq 3$ dilutions)

- Sarecycline also showed less activity against 2 Candida species

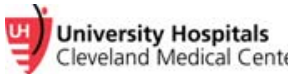

W/ SCHOOL OF MEDICINE
CASEWESTERNRESRVE
UNIVERSITY
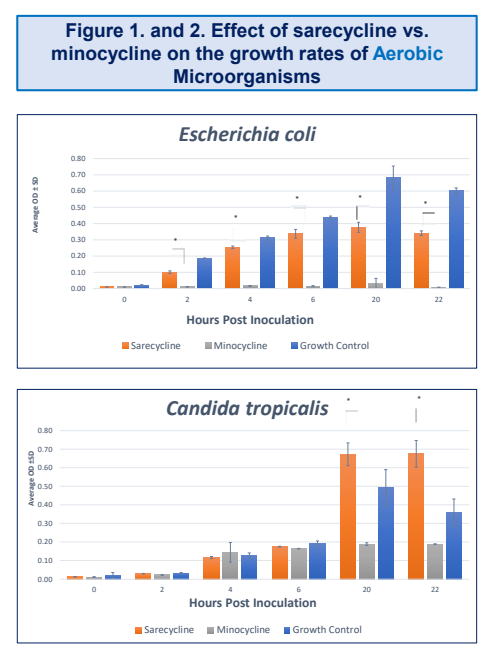

- Sarecycline showed significantly less activity against
E. coli compared to minocycline at all time points $(P$.

values $<0.05)$

- Sarecycline was significantly less active against
c. tropicalis compared to minocycline at 20 and 22
hours post exposure $(P$-values $<0.05)$ - Time kill study shows that with longer time exposur
sarecycline has less inhibibitory activity against

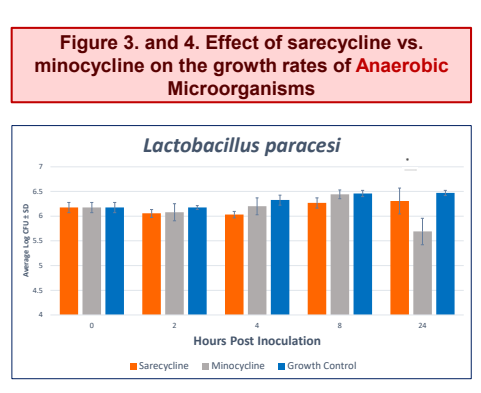

Bifidobacterium adolescentis

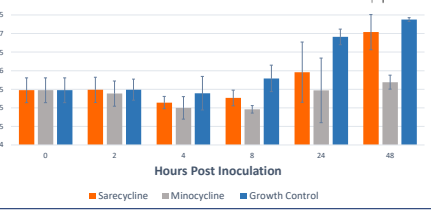

Sarecycline showed significantly less activity against L. paracasei compared ton
growth (P-value of 0.002 ) Sarecycline showed significantly less activity against
B. adolescentis compared to minocycline after 48 hours
of growth (P-value of 0.042 )

- In this study, sarecycline demonstrated less activity against $79 \%$ of the microorganisms normally found in a healthy human gut, when compared to minocycline

- Sarecycline is a narrow-spectrum antibiotic

- Our data suggests that sarecycline may have less impact on disrupting commensal and symbiotic organisms residing in the gut and is less likely to promote dysbiosis. In vivo evaluation is ongoing 